\title{
In vivo aggregation properties of the nuclear poly(A)-binding protein PABPN1
}

\author{
JOÃO PAUlO TAVANEZ, ${ }^{1}$ PATRICIA CALADO, ${ }^{1}$ JOSÉ BRAGA, ${ }^{1}$ MIGUEL LAFARGA, ${ }^{2}$ and \\ MARIA CARMO-FONSECA ${ }^{1}$ \\ ${ }^{1}$ Institute of Molecular Medicine, Faculty of Medicine, University of Lisbon, 1649-028 Lisboa, Portugal \\ ${ }^{2}$ Department of Anatomy and Cell Biology, Biomedicine Unit Associated to the CSIC, University of Cantabria, 39011 Santander, Spain
}

\begin{abstract}
A broad range of degenerative diseases is associated with intracellular inclusions formed by toxic, aggregation-prone mutant proteins. Intranuclear inclusions constitute a pathological hallmark of oculopharyngeal muscular dystrophy (OPMD), a dominantly inherited disease caused by (GCG) repeat expansions in the gene that encodes for nuclear poly(A) binding protein (PABPN1). The mutation results in an extended polyalanine stretch that has been proposed to induce protein aggregation and formation of intranuclear inclusions. Here we show that normal PABPN1 is inherently aggregation-prone when exogenously expressed in either HeLa or myogenic C2 cells. Similar deposits of insoluble PABPN1 are formed by variant forms of the protein containing either a polyalanine expansion or a complete deletion of the polyalanine tract, indicating that the mutation responsible for OPMD is not essential for formation of PABPN1 inclusions. In contrast, interfering with any of the protein domains required for stimulation of poly(A) polymerase prevents the formation of inclusions. Most surprisingly, photobleaching experiments reveal that both normal and expanded PABPN1 molecules are not irreversibly sequestered into aggregates, but rather move rapidly in and out of the inclusions. These findings have important implications for the interpretation of OPMD model systems based on exogenous expression of PABPN1.
\end{abstract}

Keywords: poly(A) binding protein; poly(A) polymerase; intranuclear inclusions; protein aggregation; oculopharyngeal muscular dystrophy

\section{INTRODUCTION}

Many neurodegenerative diseases, including Alzheimer's disease, Parkinson's disease, prion diseases, and Huntington's disease, are characterized by intracellular aggregation and deposition of pathogenic proteins (Taylor et al. 2002). In the case of Huntington and other polyglutamine diseases, a triplet repeat expansion gives rise to a mutant protein that aggregates, forming inclusion bodies that are most frequently localized in the nucleus of affected cells (for review, see Zoghbi and Orr 2000; Bossy-Wetzel et al. 2004). Expansion of a trinucleotide repeat is also the cause of oculopharyngeal muscular dystrophy or OPMD (Brais et al. 1998), a disease with poorly understood pathogenesis. The OPMD expansion is translated into a polyalanine tract in the nuclear poly $(\mathrm{A})$ binding protein $(\mathrm{PABPN} 1$ or $\mathrm{PABP} 2)$ and

Reprint requests to: Maria Carmo-Fonseca, Institute of Molecular Medicine, Faculty of Medicine, Av. Prof. Egas Moniz, 1649-028 Lisboa, Portugal; e-mail: carmo.fonseca@fm.ul.pt; fax: +351 217999412.

Article published online ahead of print. Article and publication date are at http://www.rnajournal.org/cgi/doi/10.1261/rna.7217105. because intranuclear inclusions containing PABPN1 represent a pathological hallmark of muscle cells from OPMD patients, it has been suggested that the mutated PABPN1 induces or facilitates the formation of the inclusions (Becher et al. 2000; Uyama et al. 2000; Fan et al. 2001; Bao et al. 2002; Scheuermann et al. 2003).

PABPN1 is an abundant nuclear protein that stimulates poly(A) polymerase (PAP) during polyadenylation of mRNA precursors in metazoan cells (for a recent review, see Kühn and Wahle 2004). Most eukaryotic messenger RNA (mRNA) molecules have a long tract of adenosine nucleotides at the $3^{\prime}$ end, and this so-called poly(A) tail seems to play a role in every step of the life of the mRNA. During or shortly after gene transcription, mRNA precursors are cleaved to form a new $3^{\prime}$ end that receives a tail of $\sim 250$ adenylate residues. $\operatorname{Poly}(\mathrm{A})$ addition is carried out by a poly(A) polymerase enzyme that on its own is nearly inactive (Wahle 1991a,b). In order to be active in polyadenylation, PAP depends on cleavage and polyadenylation specificity factor (CPSF), a complex of at least four polypeptides, and PABPN1. CPSF and PABPN1 are thought to cooperate in the stimulation of PAP by interacting with the enzyme 
and tethering it onto the RNA. In fact, PAP binds directly to both the $160-\mathrm{kDa}$ subunit of CPSF (Murthy and Manley 1995) and PABPN1, resulting in an increased affinity of the enzyme for RNA (Kerwitz et al. 2003). PABPN1 is also involved in controlling the length of the poly(A) to $\sim 250$ nucleotides (Wahle 1991a, 1995; Bienroth et al. 1993) and may additionally contribute to export of mRNA from the nucleus to the cytoplasm (Chen et al. 1999; Calado et al. 2000a; Bear et al. 2003).

PABPN1 is a 306-amino-acid protein with a calculated molecular mass of $32.8 \mathrm{kDa}$ that is detected as a $49-\mathrm{kDa}$ polypeptide in SDS-PAGE (Nemeth et al. 1995). The protein contains a N-terminal domain that interacts with PAP (Kerwitz et al. 2003), and two RNA binding domains required for binding to poly(A), a ribonucleoprotein-type RNA binding domain located in the middle of the protein sequence and a C-terminal domain rich in asymmetrically dimethylated arginines (Smith et al. 1999; Kühn et al. 2003).

To gain insight into the mechanisms involved in formation of OPMD nuclear inclusions, we generated green fluorescent protein (GFP) fusions with PABPN1 variants. The fusion proteins were transiently expressed in human HeLa cells and in the murine myogenic cell line, $\mathrm{C} 2$. The results show that GFP-PABPN1 aggregation occurs in the absence of polyalanine expansion. Propensity of GFP-PABPN1 to form intranuclear inclusions is coupled to stimulation of polyadenylation, and aggregation is a highly dynamic, reversible process.

\section{RESULTS}

\section{Exogenous expression of normal PABPN1 induces formation of insoluble nuclear inclusions enriched in $\operatorname{poly}(\mathrm{A}) \mathrm{RNA}$}

Immunofluorescence microscopy using affinity-purified antibodies reveals PABPN1 localized throughout the nucleoplasm with higher concentration in speckles (Krause et al. 1994). A similar distribution is observed when PABPN1 is fused to the green fluorescence protein (GFP-PABPN1) and transiently expressed in HeLa cells (Fig. 1A, arrows). However, $\sim 50 \%$ of the cells expressing GFP-PABPN1 show additional intensely labeled foci or inclusion bodies in the nucleus (Fig. 1A, arrowheads). Quantification of the ratio between the mean fluorescence intensity in these foci and in nuclear speckles indicates that GFP-PABPN1 is two- to fivefold more concentrated in the inclusions. Brightly labeled nuclear inclusions are also observed in cells expressing PABPN1 tagged with a C-terminal hemaglutinin (HA) epitope (Fig. 1B). It is therefore unlikely that formation of the inclusions is an artifact caused by the large GFP tag. Similar results were obtained upon transfection of the murine myogenic cell line C2 (Fig. 1C).

Since PABPN1 intranuclear inclusions constitute a pathological hallmark of OPMD, we thought to compare
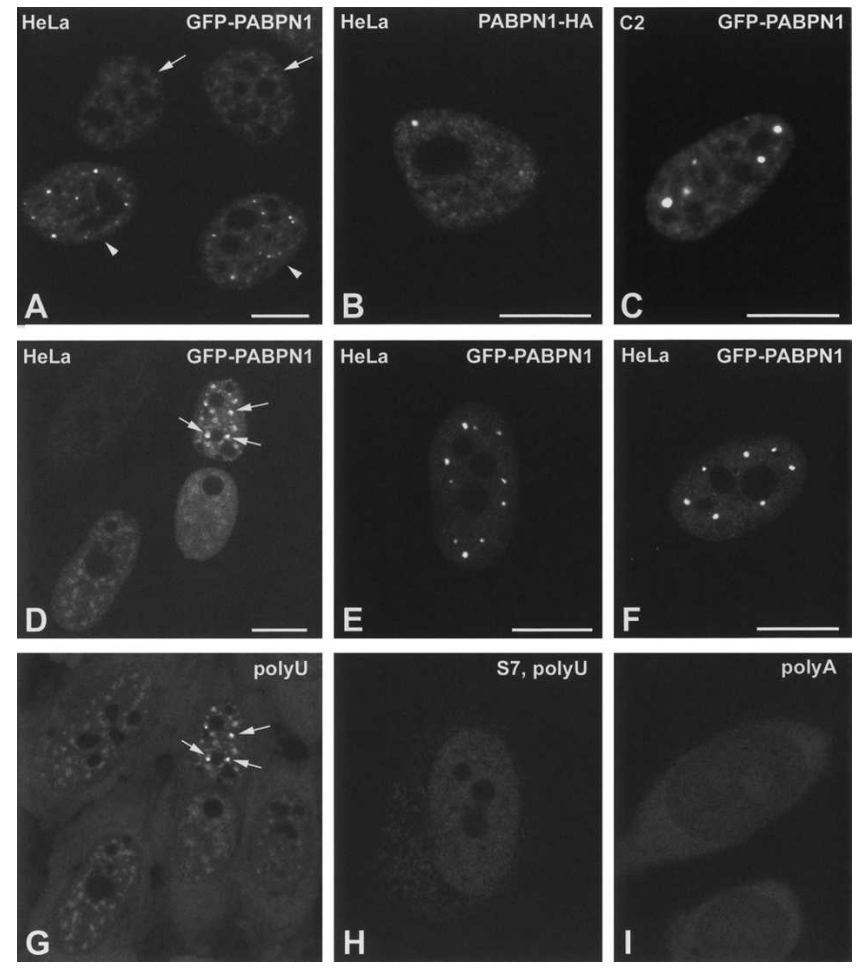

FIGURE 1. Normal PABPN1 forms intranuclear inclusions enriched in poly(A) RNA. HeLa $(A, B)$ and $\mathrm{C} 2(C)$ cells were transfected with either GFP-PABPN1 $(A, C)$ or PABPN1-HA $(B)$. A fraction of the cells $(\sim 50 \%)$ show GFP-PABPN1 distributed in the nucleoplasm with higher concentration in speckles ( $A$, arrows). Other cells show additional brightly labeled focal inclusions $(A$, arrowheads; $B, C)$. HeLa cells expressing GFP-PABPN1 were hybridized with either a $\operatorname{poly}(\mathrm{U})$ probe $(D, G ; E, H)$ or a poly $(\mathrm{A})$ probe $(F, I)$. Arrows in panels $(D, G)$ point to PABPN1 inclusions. In panels $(E, H)$, the cells were digested with S7 nuclease before hybridization. Bar, $10 \mu \mathrm{m}$.

the properties of protein deposits produced by exogenous expression of GFP-PABPN1 with those present in affected muscle fibers from OPMD patients. We had previously shown that OPMD nuclear inclusions accumulate poly(A) RNA, ubiquitin, and proteasomes (Calado et al. 2000b). To detect poly(A) RNA in cells expressing GFP-PABPN1, fluorescence in situ hybridization was performed using an oligo(U) probe (Fig. 1D,G). In nontransfected cells, as well as in cells that express GFP-PABPN1 but have no inclusions, the oligo(U) probe labels the cytoplasm and the nucleus, excluding the nucleolus. In cells that contain GFP-PABPN1 inclusions, these structures are intensely stained by the probe (Fig. 1D,G, arrows). Labeling of the GFP-PABPN1 inclusions is totally abolished when hybridization with the oligo(U) probe is performed in cells previously digested with S7 (micrococcal) nuclease (Fig. 1E,H), a 3'-OH endspecific exoribonuclease that removes poly $(\mathrm{A})$ tails (see Calado and Carmo-Fonseca 2000). Thus, the inclusions are highly enriched in poly(A) RNA. As PABPN1 binds tightly to poly(A) (Wahle 1991a; Wahle et al. 1993), we incubated cells with an oligo(A) probe (Fig. 1F,I). No specific labeling is observed, suggesting that most of the GFP-PABPN1 pres- 
A

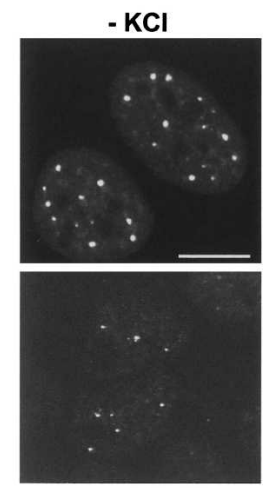

$+1 \mathrm{M} \mathrm{KCl}$

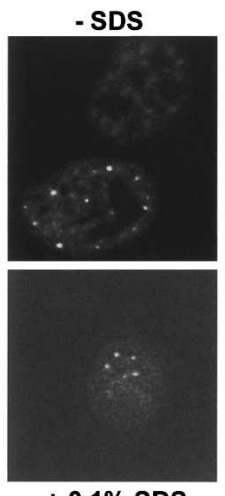

$+0.1 \%$ SDS

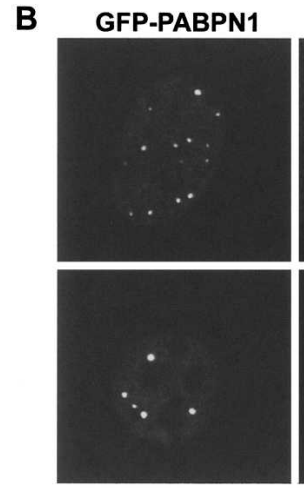

GFP-PABPN1
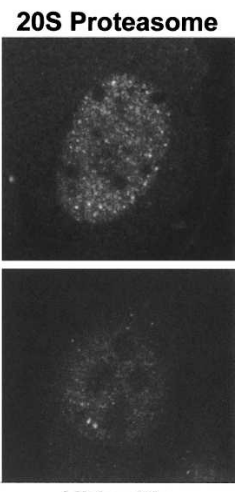

Ubiquitin

FIGURE 2. Normal PABPN1 forms insoluble inclusions that fail to recruit ubiquitin and proteasomes. (A) HeLa cells expressing GFP-PABPN1 were treated with $1 \mathrm{M} \mathrm{KCl}$ or $0.1 \%$ SDS. Note that GFP-PABPN1 is completely extracted from the nuclear speckles, whereas the protein in focal inclusions is insoluble. (B) HeLa cells expressing GFP-PABPN1 were immunolabeled with antibodies directed against $20 \mathrm{~S}$ proteasomes and ubiquitin. Bar, $10 \mu \mathrm{m}$.

ent in the inclusions is associated with nuclear poly(A) RNA.

Next, we tested whether the GFP-PABPN1 nuclear inclusions contain insoluble protein and recruit proteasomes (Fig. 2). As pathological OPMD inclusions are resistant to salt extraction (Calado et al. 2000b), we treated HeLa cells expressing GFP-PABPN1 with either $1 \mathrm{M} \mathrm{KCl}$ or $0.1 \%$ SDS. As depicted in Figure 2A, the inclusions remain brightly stained, whereas most of the fluorescence disappeared from the nuclear speckles. In addition, HeLa cells expressing GFP-PABPN1 were immunolabeled with antibodies directed against $20 \mathrm{~S}$ proteasomes and ubiquitin (Fig. 2B). As previously described, HeLa cells contain nuclear foci, termed clastosomes, that are enriched in ubiquitin conjugates and the proteolytically active $20 \mathrm{~S}$ core of the proteasome (Lafarga et al. 2002). However, neither ubiquitin nor proteasomes are detected in the GFP-PABPN1 inclusions.

Viewed with the electron microscope, the OPMD nuclear inclusions consist of collections of tubular filaments $\sim 8.5 \mathrm{~nm}$ in external diameter, disposed in tangles or palisades decorated by anti-PABPN1 antibodies (Calado et al. 2000b). To unambiguously determine the structure of the inclusions induced by GFP-PABPN1 overexpression, immunoelectron microscopy was performed using anti-GFP antibodies (Fig. 3). Although the gold particles are predominantly associated with amorphous material, a few scattered tubular filaments with an external diameter of $\sim 12 \mathrm{~nm}$ can be identified (Fig. 3, arrows in a,b). Clearly, the GFPPABPN1 inclusions are much less orga-
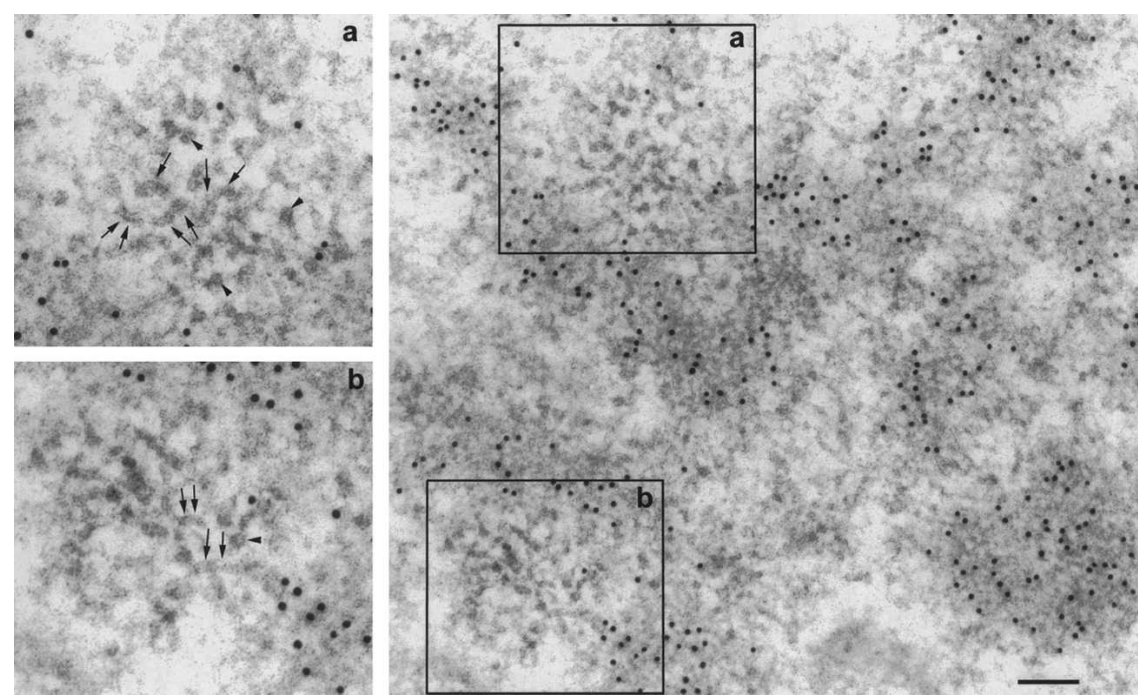

FIGURE 3. Inclusions formed by normal PABPN1 contain disorganized filaments. Thin sections of C2 cells expressing GFP-PABPN1 were immuno-gold labeled with anti-GFP antibody. The inclusions are largely amorphous, but contain a few short filaments. Panels on the left represent a high magnification of areas $(a)$ and $(b)$. Filaments are indicated by arrows (longitudinal section) and arrowheads (transverse section). Bar, $100 \mathrm{~nm}$. 


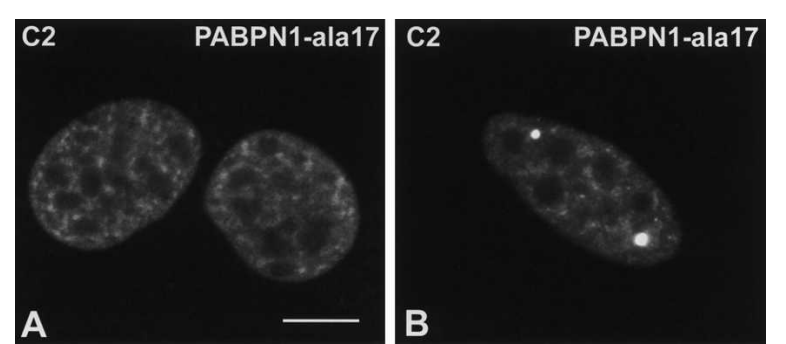

FIGURE 4. The distribution of expanded PABPN1 is similar to the normal protein. C2 cells expressing GFP-PABPN1-ala17, a plasmid that encodes a PABPN1 mutant with a stretch of 17 alanines instead of $10(A, B)$. Bar, $10 \mu \mathrm{m}$.

In conclusion, our results indicate that exogenous expression of normal PABPN1 is sufficient to induce the formation of insoluble intranuclear inclusions that are highly enriched in poly(A) RNA. Moreover, expression of either wild-type or expanded PABPN1 leads to the formation of intranuclear inclusions with similar properties. Thus, expansion of the polyalanine homopolymer caused by the OPMD mutation is not required to induce PABPN1 aggregation in vivo.

\section{Poly(A) polymerase is recruited to PABPN1 inclusions}

Having shown that nuclear inclusions induced by exogenous expression of PABPN1 are enriched in poly(A) RNA, we asked whether specific mRNAs are sequestered by these structures. Fluorescence in situ hybrization (FISH) was therefore performed using a probe to the abundant $\beta$-actin mRNA. Although fluorescent focal signals corresponding to nascent $\beta$-actin transcripts were observed in the nucleus, no labeling was detected in the GFP-PABPN1 inclusions (Fig. $6 \mathrm{~A})$. Similar results were obtained using a probe to detect the abundant GFP fusion transcripts expressed in transfected cells. Possible explanations for these negative results could be that the local concentration of actin or GFP-PABPN1 mRNAs in the inclusions is too low to be detected by FISH, or that the inclusions sequester a particular subset of mRNAs. Alternatively, we may consider that mRNAs are sequestered in the inclusions but are mostly degraded while the poly(A) tails persist, or that the poly(A) RNA present in the inclusions does not correspond to mRNA.

To further investigate whether the PABPN1 nuclear inclusions sequester other proteins involved in mRNA biogenesis, HeLa cells expressing either GFP-PABPN1 or GFP-PABPN1-ala17 were immunolabeled with antibodies directed against hnRNP proteins, splicing factors, exon junction complex proteins, $3^{\prime}$-end processing factors, and mRNA export factors (Fig. 6B). Antibodies directed against hnRNP A1 and C proteins, splicing factor SC-35, exon junction components REF and DEK, the export factor TAP and the cytoplasmic poly(A) binding protein (PABPC) failed to label either the GFP-PABPN1 or the GFPPABPN1-ala17 inclusions. Additionally, immunolabeling experiments were performed with antibodies against the cleavage and polyadenylation specificity factor subunits CPSF-30, CPSF-70, CPSF-100, and CPSF-160, and the cleavage stimulation factor subunit Cstf-50. Neither of these proteins was detected in the GFP-PABPN1 or GFPPABPN1-ala17 inclusions. In contrast, an antibody specific for poly(A) polymerase (PAP) reveals colocalization with the aggregates (Fig. 7). In cells that do not contain GFPPABPN1 inclusions, PAP is uniformly distributed throughout the nucleus and the cytoplasm (Fig. $7 \mathrm{~A}, \mathrm{E}$ ), whereas in cells that show GFP-PABPN1 or GFP-PABPN1-ala17 nuclear inclusions, PAP appears enriched in these structures (Fig. 7 B,F). The distribution of PAP is not altered in cells that express a point mutant version of PABPN1 that does not form inclusions in the nucleus (GFP-PABPN1-dm, see below), indicating that anti-PAP antibodies are not crossreacting with the fusion protein (Fig. 7 C,G). Labeling of the PABPN1 inclusions by anti-PAP antibodies resisted extraction with $1 \mathrm{M} \mathrm{KCl}$, suggesting that $\mathrm{PAP}$ is tightly bound to these structures (Fig. 7 D,H).

\section{PABPN1 domains required for aggregation in vivo}

To investigate how the structure of PABPN1 influences aggregation and formation of nuclear inclusions, we generated GFP fusions to mutant forms of the protein. For each mu-
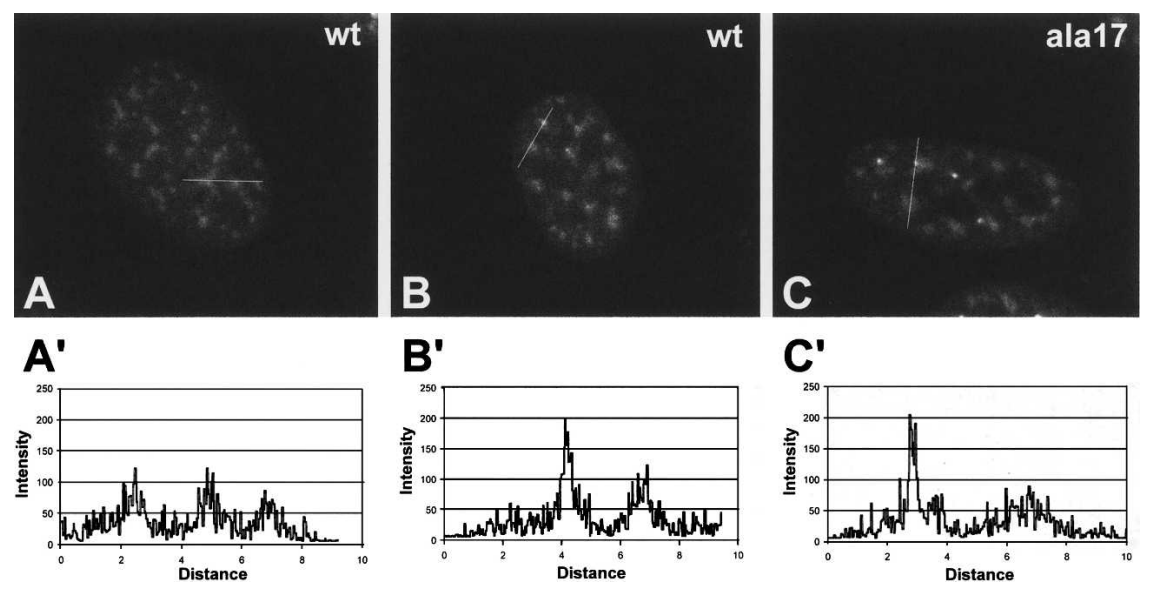

FIGURE 5. Normal and expanded GFP-PABPN1 concentrate similarly in nuclear inclusions. HeLa cells expressing wild-type GFP-PABPN1 $(A, B)$ or GFP-PABPN1-ala17 $(C)$. The graphs in $\left(A^{\prime}-C^{\prime}\right)$ correspond to a quantitative measurement of fluorescence intensity along the line indicated in the images above. The average ratio between the fluorescence intensity in the smallest visible nuclear inclusions and the surrounding nuclear speckles in cells expressing normal or expanded GFP-PABPN1 is 1.8 and 1.9 , respectively. 
A
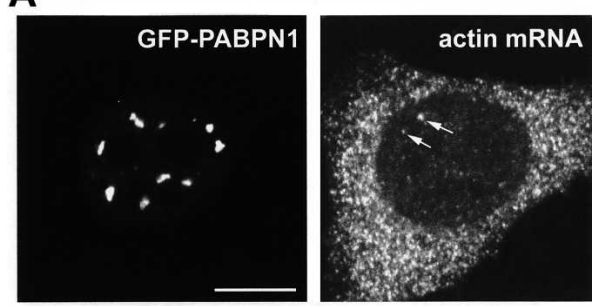

B
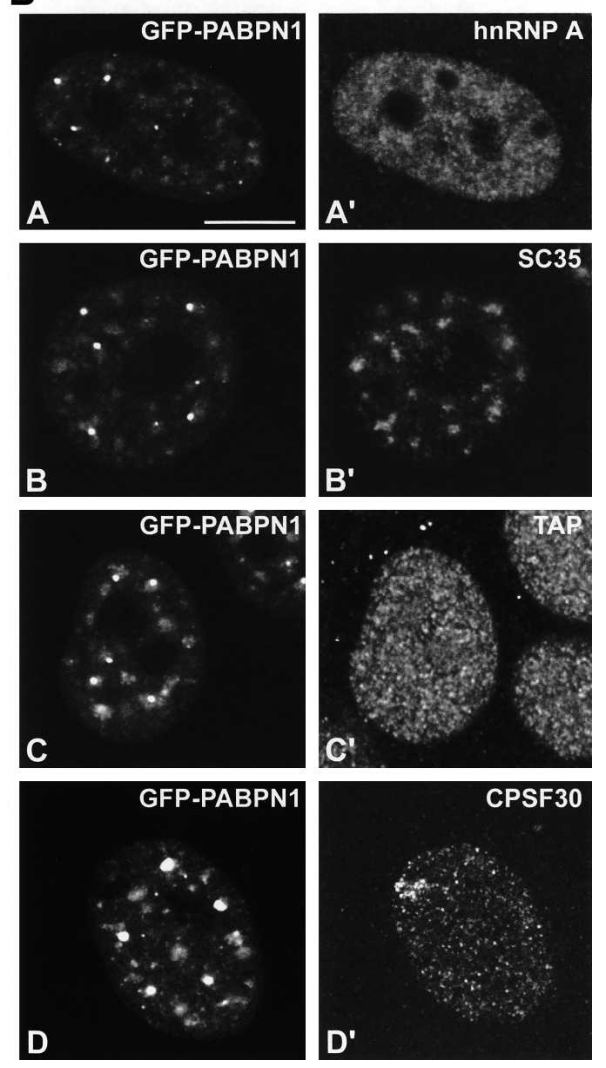

FIGURE 6. PABPN1 inclusions do not sequester mRNA. (A) HeLa cells expressing GFP-PABPN1 were hybridized with a probe specific for $\beta$-actin mRNA. Arrows point to nascent actin transcripts in the nucleus. (B) HeLa cells expressing GFP-PABPN1 were immunolabeled with antibodies against hnRNPA1, SC35, TAP, and CPSF-30. Bar, $10 \mu \mathrm{m}$.

tant, we analyzed HeLa cells with mean fluorescence intensity similar to that of cells that do not contain GFPPABPN1 inclusions (Figs. 8, 9, low) and cells with mean fluorescence intensity at least threefold higher (Figs. 8, 9, high). Integrity of each fusion protein was confirmed by Western blotting (Figs. 8, 9).

The primary structure of PABPN1 suggests a separation into three domains (Kühn et al. 2003). The acidic N-terminal domain includes the polyalanine homopolymer and is essential for the stimulation of $\operatorname{poly}(\mathrm{A})$ polymerase. The RNP-type domain, which extends from Met ${ }^{161}$ to $\mathrm{Thr}^{257}$ is essential but not sufficient for poly(A) binding. The dimethylated-arginine-rich C-terminal domain starts at $\mathrm{Asp}^{258}$ and is required for full poly(A) affinity.
First, we studied the influence of the polyalanine homopolymer on PABPN1 aggregation. The panels in Figure 8A depict cells expressing normal PABPN1, an expansion mutant with a stretch of 17 alanines instead of 10 (GFPPABPN1-ala17), and a deletion mutant devoid of the homopolymer (GFP-PABPN1- $\Delta$ ala). The three distinct protein variants show a similar nuclear distribution, i.e., concentration in nuclear speckles with additional accumulation in nuclear inclusions. These results argue that the $\mathrm{N}$-terminal polyalanine stretch is dispensable for nuclear inclusion formation.

To investigate the role of RNA binding in protein aggregation (Fig. 8B), we analyzed cells expressing a PABPN1 variant containing two point mutations at $\mathrm{Y} 175 \mathrm{~A}$ and F215A (GFP-PABPN1-dm), a combination of this form with the alanine expansion (GFP-PABPN1-ala17dm) and a complete C-terminal deletion mutant (GFP-PABPN1$\Delta$ C49). The PABPN1-dm and PABPN1- $\Delta$ C49 mutants have strongly reduced binding to poly(A) (Kühn et al. 2003) and are both defective in the stimulation of polyadenylation (Kerwitz et al. 2003). In contrast to normal PABPN1, the three mutants analyzed in Figure $8 \mathrm{~B}$ remain uniformly distributed throughout the nucleoplasm. These mutant proteins do not localize to nuclear speckles, do not form inclusions, and are completely solubilized when cells are extracted with $0.1 \%$ Triton-X100 prior to fixation.

In a phylogenetic comparison, the PABPN1 N-terminal domain contains a highly conserved sequence of 30 amino acids (L119-Q147) that is predicted to form an amphipathic $\alpha$-helix or coiled-coil domain (Kerwitz et al. 2003; Kühn et al. 2003). Expression of a series of PABPN1 variants with deletion of residues 1-50 (GFP-PABPN1- $\Delta$ N50), 1-65 (GFP-PABPN1- $\Delta$ N65), and 1-113 (GFP-PABPN1- $\Delta$ N113) shows that all these mutants form intranuclear inclusions (Fig. 9A; data not shown). In contrast, deletion of residues 1-160 (GFP-PABPN1- $\Delta$ N160) prevents the formation of inclusions (Fig. 9A). Expression of an additional variant with deletion of residues 118-145 (GFP-PABPN1- $\Delta 118-$ 145) similarly fails to form inclusions, suggesting an involvement of the putative $\alpha$-helix in PABPN1 aggregation (Fig. 9A). To further investigate this idea, PABPN1 variants containing point mutations in the predicted $\alpha$-helical domain were expressed as GFP fusion proteins and tested for aggregation. The mutants L119A, A133S, and V143A have a nuclear distribution similar to wild-type PABPN1. In contrast, an alanine substitution of L136, which abolishes PABPN1 stimulation of poly(A) polymerase (Kerwitz et al. 2003), produces an uniform distribution of the protein throughout the nucleoplasm (Fig. 9B). Although the vast majority of cells expressing GFP-PABPN1-L136A are devoid of intranuclear inclusions, $2 \%-5 \%$ of the cells do contain aggregates.

Taken together these results suggest that formation of PABPN1 inclusions is functionally coupled to polyadenylation. 


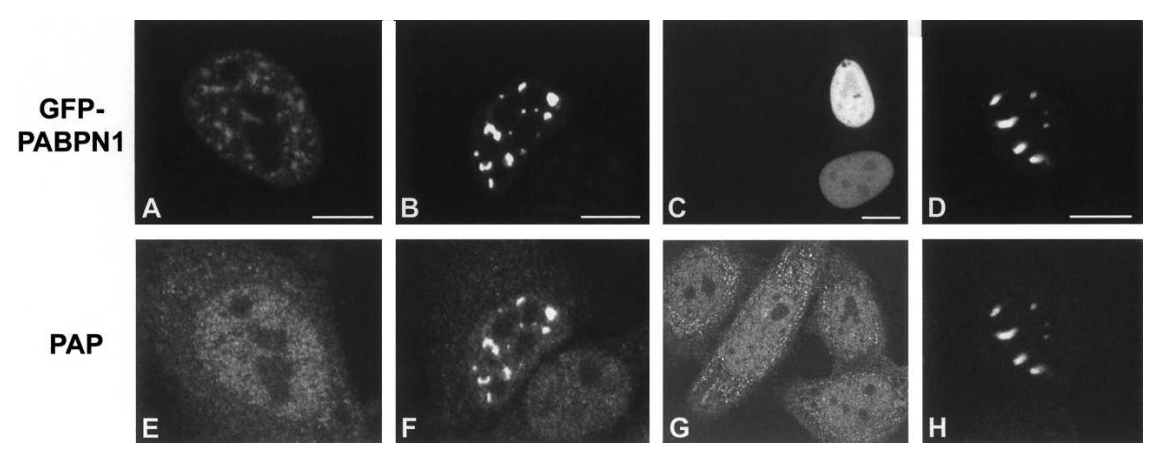

FIGURE 7. PABPN1 inclusions recruit PAP. HeLa cells expressing wild-type GFP-PABPN1 $(A, B, D)$ or GFP-PABPN1-dm $(C)$ were immunolabeled with antibodies against poly(A) polymerase, PAP $(E-H)$. In panels $(D, H)$ the cells were extracted with $1 \mathrm{M} \mathrm{KCl}$. Bar, $10 \mu \mathrm{m}$.

\section{PABPN1 molecules in nuclear inclusions are in constant exchange with the nucleoplasmic pool}

Intracellular inclusions formed by aggregation-prone proteins represent a hallmark of several degenerative diseases, and one of the mechanisms proposed for toxicity of these pathological inclusions is the sequestration of critical factors by the aggregated protein (for review, see Taylor et al. 2002). The finding that many pathological inclusions are highly insoluble further supports the view that abnormal proteins are responsible for the formation of stable structures capable of trapping essential cellular components. Similarly to pathological inclusions, the GFP-PABPN1 inclusions are resistant to detergent and high salt extraction. We therefore expected that these structures correspond to stable, static protein deposits in the nucleus. To directly monitor the dynamics of PABPN1 interactions within the inclusions, photobleaching analysis was performed in live HeLa cells (Fig. 10). In photobleaching, a small region in the nucleus is illuminated at high intensity by a high-powered focused laser beam. As a consequence, GFP molecules present within that region are irreversibly bleached without detectable damage to the cell. Subsequent diffusion of bleached GFP molecules out of the bleached area and diffusion of surrounding nonbleached GFP molecules into the bleached area leads to a recovery of fluorescence, which is recorded at low laser power. There are two variations of photobleaching experiments that yield different types of information: fluorescence recovery after photobleaching (FRAP), and fluorescence loss in photobleaching (FLIP). In FRAP, a region is bleached once and subsequent recovery of fluorescence in the bleached area is monitored over time. In FLIP, a region is repeatedly bleached, and the loss of fluorescence from the surrounding nonbleached area is monitored over time (for review, see White and Stelzer 1999; Reits and Neefjes 2001).

As depicted in Figure 10A, after bleaching of an entire inclusion, GFP fluorescence from the nucleoplasmic pool rapidly reassociated. Figure 10B shows the recovery of relative fluorescence intensity within the bleach region plotted PABPN1 with an amino-terminal fragment of the Cajal body protein $\mathrm{p} 80$ coilin remains essentially immobile, as previously described (Calapez et al. 2002).

To determine the dissociation kinetics of GFP-PABPN1 from the inclusions, we performed FLIP (Fig. 10C). By repeatedly bleaching a nucleoplasmic area, $>95 \%$ of GFP fluorescence was lost from all inclusion bodies. We therefore conclude that the pool of GFP-PABPN1 molecules in the inclusions is continuously and rapidly exchanged with the nucleoplasmic pool.

\section{DISCUSSION}

Here we show that normal PABPN1 tends to aggregate and form intranuclear deposits when exogenously expressed in either muscle or nonmuscle cells. Similarly to the intranuclear inclusions present in affected muscle from OPMD patients, the PABPN1 deposits are highly enriched in poly(A) RNA and resist high-salt extraction. These deposits are, however, much less organized at the ultrastructural level than the disease inclusions and fail to concentrate ubiquitin and proteasomes. Thus, exogenous expression of PABPN1 mimics some, but not all, of the pathological features detected in OPMD muscle biopsies.

Expression analysis of PABPN1 variants containing either a polyalanine expansion or a complete deletion of the polyalanine tract, unambiguously demonstrates that expansion of the polyalanine homopolymer caused by the OPMD mutation is not required to induce PABPN1 aggregation and formation of nuclear inclusions. In agreement with this observation, we had previously described the presence of filamentous intranuclear inclusions composed of wild-type PABPN1, poly(A) RNA, ubiquitin, and proteasomes in normal hypothalamic secretory neurons (Berciano et al. 2004). The ubiquitin-proteasome system plays a key role in clearing unwanted proteins from the cell, and its presence in most disease-related inclusions most likely reflects a cellular response to excess abnormal protein (for review, see Orr 2001). The proteasome is a multimeric enzyme composed of two subcomplexes, the $20 \mathrm{~S}$ proteolytic core and the $19 \mathrm{~S}$ 


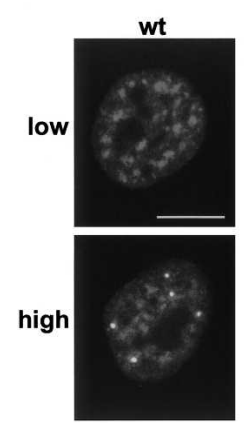

ala17

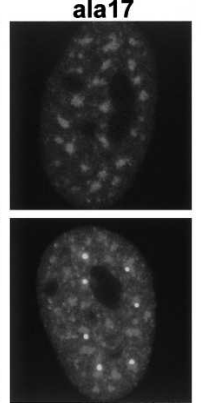

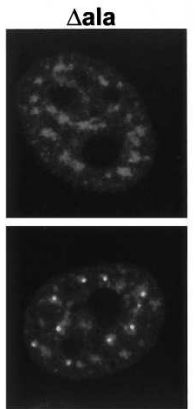

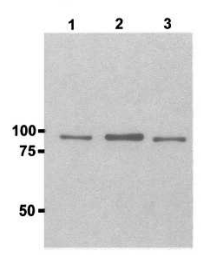

\section{B}
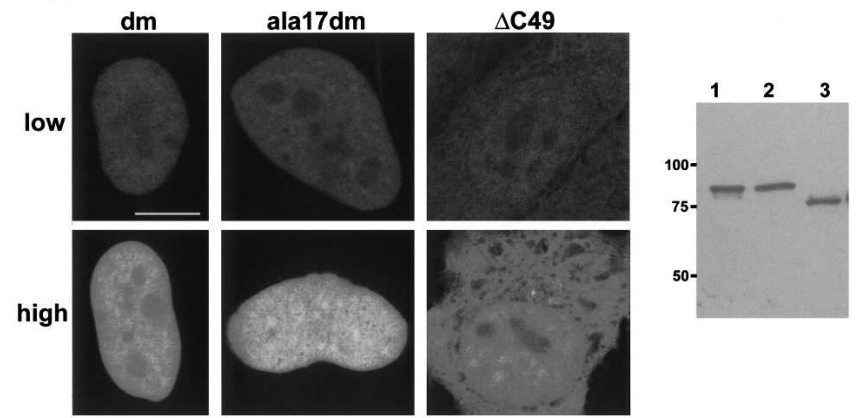

FIGURE 8. Formation of PABPN1 inclusions requires binding to poly(A). HeLa cells were transfected with plasmids encoding PABPN1 variants fused to GFP. For each PABPN1 variant, the upper panels depict cells with mean fluorescence intensity similar to that of cells that do not contain GFP-PABPN1-wt inclusions (low) and cells with mean fluorescence intensity at least threefold higher (high). Bar, $10 \mu \mathrm{m}$. (A) HeLa cells expressing normal PABPN1 (GFP-PABPN1-wt), an expansion mutant with a stretch of 17 alanines instead of 10 (GFP-PABPN1-ala17), and a deletion mutant devoid of the homopolymer (GFP-PABPN1- $\Delta$ ala). Western blot analysis of each fusion protein is shown in lanes $1,2,3$, respectively. Molecular weight markers $(\mathrm{kDa})$ are indicated on the left. $(B)$ HeLa cells expressing a PABPN1 variant containing two point mutations at Y175A and F215A (GFP-PABPN1-dm), a combination of this form with the alanine expansion (GFP-PABPN1-ala17dm) and a complete C-terminal deletion mutant (GFP-PABPN1- $\Delta$ C49). Western blot analysis of each fusion protein is shown in lanes $1,2,3$, respectively. Molecular weight markers $(\mathrm{kDa})$ are indicated on the left.

regulator. Protein substrates degraded by the proteasome must first be marked by covalent ligation to ubiquitin in order to become targeted for proteolysis (Bochtler et al. 1999). The finding that the inclusion bodies in secretory neurons contain ubiquitin and proteasomes (Berciano et al. 2004) suggest that under some circumstances, deposition of wild-type PABPN1 activates the cellular mechanism responsible for disposal of abnormal proteins. In contrast, absence of ubiquitin and proteasomes from the inclusions induced by transient expression of wild-type PABPN1 may indicate that formation of these deposits is entirely a passive massaction driven process with self-assembly of excess monomers into a growing aggregate. In this regard it is interesting to note that the smallest visible GFP-PABPN1 inclusions are consistently observed in close proximity to nuclear speckles (Fig. 5). As nuclear speckles contain the highest concentration of PABPN1 in normal nuclei, these compartments may act as nucleation sites for protein aggregation.

A detailed analysis of deletion and point mutant variants of PABPN1 shows that formation of intranuclear deposits is tightly coupled to the stimulatory activity of the protein on polyadenylation. In fact, an important role of PABPN1 in the nucleus is to stimulate poly $(\mathrm{A})$ polymerase (PAP) by recruiting the enzyme to the substrate RNA (Kerwitz et al. 2003). Recruitment involves a direct interaction of PAP with a coiled-coil structure within the $\mathrm{N}$-terminal domain
A

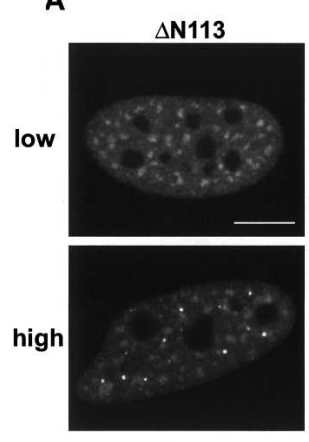

B

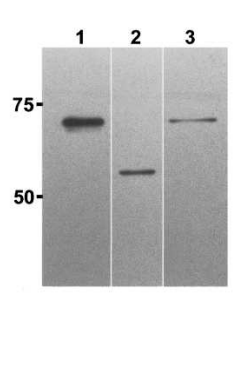

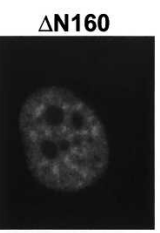

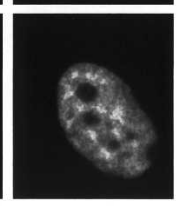

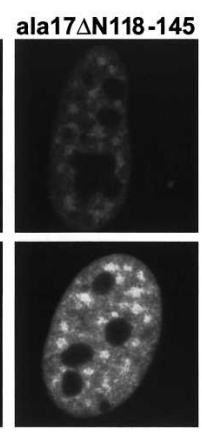

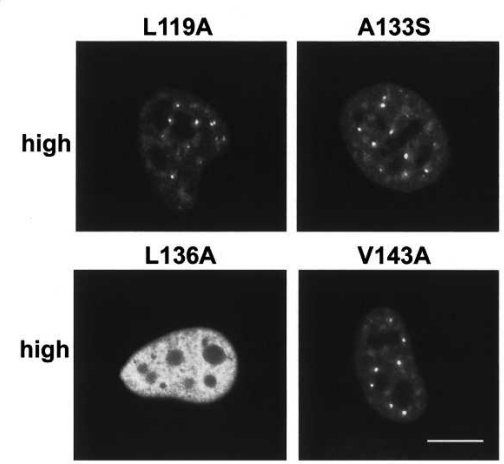

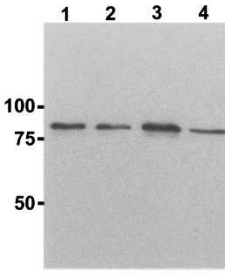

FIGURE 9. Formation of PABPN1 inclusions requires the coiled-coil domain. HeLa cells were transfected with plasmids encoding PABPN1 variants fused to GFP. (A) HeLa cells expressing PABPN1 variants containing deletions of amino acids 1-113 (GFP-PABPN1- $\Delta$ N113), 1-160 (GFP-PABPN1- $\Delta$ N160) and 118-145 (GFP-PABPN1- $1118-145)$. For each PABPN1 variant, the upper panels depict cells with mean fluorescence intensity similar to that of cells that do not contain GFP-PABPN1-wt inclusions (low) and cells with mean fluorescence intensity at least threefold higher (high). Western blot analysis of each fusion protein is shown in lanes 1,2,3, respectively. Molecular weight markers ( $\mathrm{kDa}$ ) are indicated on the left. (B) HeLa cells expressing PABPN1-ala17 substitution mutants L119A, A133S, L136A, and V143A (only cells with high fluorescence intensity are shown). Bar, $10 \mu \mathrm{m}$. Western blot analysis of each fusion protein is shown in lanes $1,2,3$, respectively, and molecular weight markers $(\mathrm{kDa})$ are indicated on the left. 


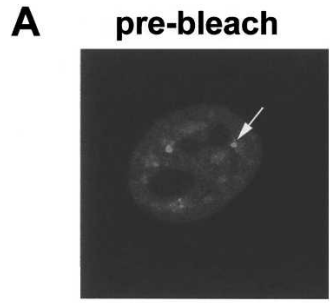

C

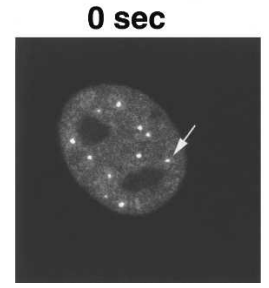

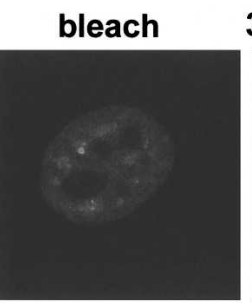

$4.8 \mathrm{sec}$

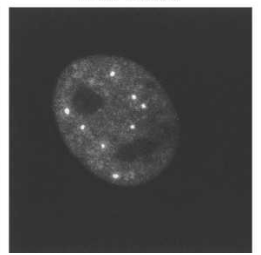

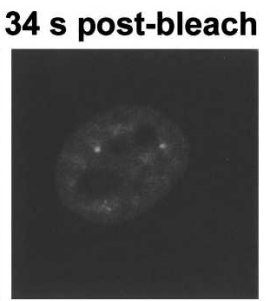

$154.8 \mathrm{sec}$

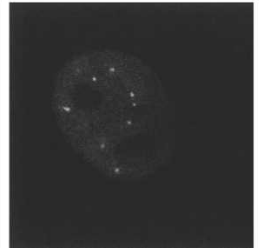

B

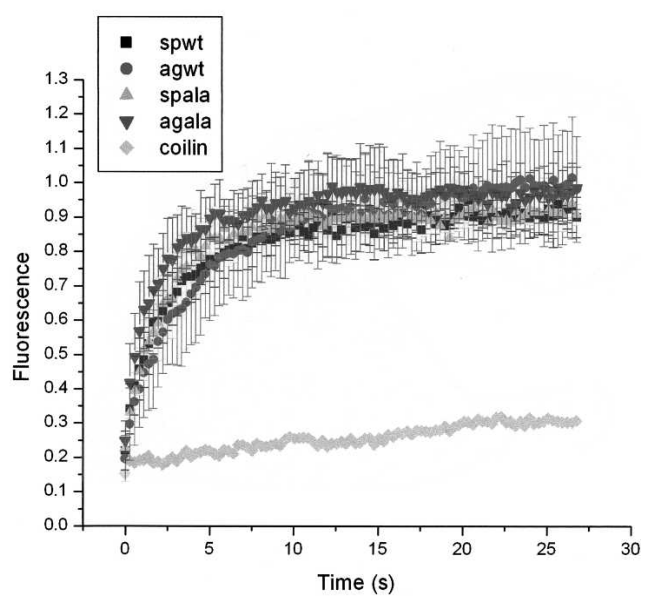

FIGURE 10. PABPN1 molecules are not irreversibly sequestered into nuclear inclusions. (A) FRAP sequence of a HeLa cell expressing GFPPABPN1wt. The arrow indicates the bleached region. (B) FRAP kinetics of cells expressing GFP-PABPN1wt, GFP-PABPN1-17ala, and GFPPABPN1 fused to an N-terminal fragment of p80 coilin (amino acids 1-468). The bleached region contained either an aggregate (ag) or a nuclear speckle (sp). The recovery curves correspond to a pool of three independent experiments, with 10 different cells analyzed per experiment. Error bars represent standard deviations. $D$ values represent mean \pm SEM. (C) FLIP sequence of a HeLa cell expressing GFP-PABPN1wt. Repetitive bleaching was performed in the area indicated by the arrow.

of PABPN1. In addition, RNA allosterically activates the interaction between the two proteins, and therefore the stimulatory effect depends on binding to RNA. Physiological binding of PABPN1 to poly(A) RNA requires both the arginine-rich C-terminal domain, and a ribonucleoproteintype RNA binding domain (RNP domain) located approximately in the middle of the protein (Kühn et al. 2003). Deletion of either the C-terminal domain of PABPN1 or substitution of two essential amino acid residues in the RNP domain completely abolishes aggregation. Likewise, deletion of the 30 amino acid (L119-Q147) coiled-coil domain or substitution of L136, which abolishes stimulation of polyadenylation (Kerwitz et al. 2003), prevented formation of PABPN1 insoluble deposits. Thus, interfering with any of the PABPN1 domains involved in stimulation of polyadenylation abolishes formation of intranuclear inclusions.

In further agreement with the view that PABPN1 aggregation is coupled to polyadenylation, the PABPN1 inclusions are highly enriched in poly(A) RNA and PAP. The inclusions are not labeled by an oligo(A) probe, arguing that most, if not all, PABPN1 is associated with nuclear poly(A) RNA. Intriguingly, no specific cellular mRNA or proteins known to associate with mRNAs were detected accumulated in the nucleus, raising the possibility that the nuclear poly(A) enriched in the PABPN1 inclusions does not correspond to mRNA. It is noteworthy that the nature of poly(A) RNA present in nuclear speckles also remains elusive (for review, see Lamond and Spector 2003). Assuming that PABPN1 aggregates arise from nuclear speckles, it is likely that speckles and inclusions may contain the same type of poly(A) RNA.

Unexpectedly, the results from photobleaching experiments indicate that PABPN1 molecules are not irreversibly sequestered into aggregates, but rather move rapidly in and out of the insoluble deposits. This finding clearly contrasts with previous reports demonstrating that GFP fusions to polyglutamine expansions localized to nuclear inclusions are immobilized there (Chai et al. 2002; Kim et al. 2002). FRAP and FLIP analysis of expanded polyglutamines (Kim et al. 2002) or expanded polyglutamine proteins (Chai et al. 2002) reveal that these proteins have significantly reduced mobility in the inclusions, whereas nonmutant forms of the proteins do not aggregate. These studies further showed immobilization of TBP and CBP present in the inclusions, supporting a model of disease in which coaggregation and sequestration of transcription factors contributes to pathogenesis. Our FRAP and FLIP results reveal that both nonexpanded (wild-type) and expanded PABPN1 are highly mobile proteins in the nucleus, irrespective of the presence of nuclear inclusions. This lack of immobilization argues that, despite being resistant to high-salt extraction, the PABPN1 inclusions represent storage sites of concentrated protein but not irreversible aggregates. A pool of polyglutamine-expanded ataxin1 that rapidly exchanges between nuclear inclusions and the nucleoplasm has also been detected by photobleaching experiments (Stenoien et al. 2002). Thus, our results reinforce the view that not all nuclear inclusions are necessarily static structures that could contribute to pathogenesis by irreversibly trapping other nuclear components.

At present we cannot determine in what extent the observed GFP-PABPN1 aggregates reproduce the pathological process that occurs in the nuclei of muscle cells from OPMD patients. As OPMD cells containing nuclear inclusions fail to grow in culture, current efforts are aimed at constructing alternative model systems for this disease. By 
providing a detailed characterization of the aggregation properties of exogenously expressed PABPN1, our results contribute a framework for improved design of OPMD models.

\section{MATERIALS AND METHODS}

\section{Cell culture, immunofluorescence, and in situ hybridization}

HeLa cells (derived from human cervical cancer) and C2 cells (an established murine myogenic cell line derived from perinatal mouse skeletal muscle) were used in this study.

HeLa and C2 cells were cultured as monolayers in Modified Eagle's Medium (MEM, Gibco Life Technologies) and Dulbecco's Modified Eagle's Medium (DMEM, Gibco), respectively. MEM was supplemented with $10 \%$ Fetal Calf Serum (FCS, Invitrogen) and $1 \%$ nonessential amino acids (Gibco), whereas DMEM was supplemented with $20 \%$ FCS.

Cells grown on $10 \times 10 \mathrm{~mm}$ coverslips were briefly rinsed in phosphate buffered saline (PBS) and fixed in 3.7\% formaldehyde (freshly prepared from paraformaldehyde) diluted in PBS for 10 min at room temperature. Cells were then permeabilized with $0.5 \%$ Triton X-100 in PBS for $10 \mathrm{~min}$ at room temperature. Alternatively, cells were incubated with $0.1 \%$ Triton X-100 in CSK buffer $(100 \mathrm{mM} \mathrm{NaCl}, 300 \mathrm{mM}$ sucrose, $10 \mathrm{mM}$ PIPES, $3 \mathrm{mM}$ $\mathrm{MgCl}_{2}, 1 \mathrm{mM}$ EGTA, $\mathrm{pH}$ 6.8) for $5 \mathrm{~min}$ on ice prior to fixation. Cells were additionally extracted with either $0.1 \%$ Triton X-100, $1 \mathrm{M} \mathrm{KCl}$ in CSK buffer for $5 \mathrm{~min}$ on ice, or $0.1 \%$ SDS in HPEM buffer (30 mM Hepes, $65 \mathrm{mM}$ Pipes, $10 \mathrm{mM}$ EGTA, $2 \mathrm{mM} \mathrm{MgCl}$, $\mathrm{pH}$ 6.9) for $3 \mathrm{~min}$ at room temperature, followed by formaldehyde fixation.

For immunofluorescence, cells were rinsed in PBS containing $0.05 \%$ Tween 20 (PBS-Tw), incubated for $1 \mathrm{hr}$ with primary antibodies diluted in PBS-Tw, washed with PBS-Tw and incubated with appropriate secondary antibodies for $30 \mathrm{~min}$. After washing with PBS-Tw, the samples were mounted in VectaShield (Vector Laboratories Inc.) and sealed with nail polish. Secondary antibodies conjugated to FITC or Texas Red were obtained from Jackson ImmunoResearch Laboratories. The following primary antibodies were used in this study: monoclonal antibodies $4 \mathrm{~F} 4$ and $9 \mathrm{H} 10$ against hnRNPC and hnRNP Al proteins, respectively (Choi and Dreyfuss 1984); monoclonal antibody against the cytoplasmic poly(A)-binding protein, PABPC (Görlach et al. 1994); monoclonal antibody anti-SC35 (Fu and Maniatis 1990); monoclonal antibodies against CPSF-160 and CPSF-100 (Jenny et al. 1994), CstF-50 (Takagaki et al. 1990); rabbit polyclonal antibodies specific for poly(A) polymerase, CPSF-73, and CPSF-30 (Barabino et al. 1997), TAP/NXF1 (Braun et al. 1999), REF (Rodrigues et al. 2001), and DEK (Fornerod et al. 1995). Proteasomes were detected using polyclonal antibodies directed against the $20 \mathrm{~S}$ catalytic core (Arribas et al. 1994; Mengual et al. 1996) and ubiquitin-conjugates were labeled with antibody Z0458 from DAKO. The hemaglutinin epitope was detected with monoclonal antibody anti-HA from COVANCE.

In situ hybridization to detect polyA RNA was performed as previously described (Gama-Carvalho et al. 1997). In situ hybridization to detect $\beta$-actin mRNA was performed using as probe, a plasmid that contains full length $\beta$-actin cDNA (Lennon et al.
1996). The plasmid was labeled with digoxigenin-11-dUTP (Roche Biochemicals) by nick translation (Johnson et al. 1991) and hybridization was performed according to Zirbel et al. (1993). Prior to hybridization, cells were rinsed once in PBS, fixed in 3.7\% formaldehyde in PBS for $10 \mathrm{~min}$ at room temperature and permeabilized with $0.5 \%$ Triton X-100 and $0.5 \%$ Saponin in PBS for $10 \mathrm{~min}$ at room temperature. The cells were then incubated in a 2X SSC solution, at $37^{\circ} \mathrm{C}$, for $5 \mathrm{~min}$ and the probe was added at a final concentration of $10 \mathrm{ng} / \mu \mathrm{l}$. Hybridization occurred overnight at $37^{\circ} \mathrm{C}$ in a moist chamber. The probe was detected using Cy3 anti-Dig antibody (Jackson ImmunoReseach Laboratories).

\section{Construction and expression of fusion proteins}

The GFP-PABPN1-wt, GFP-PABPN1-dm, GFP-PABPN1- $\Delta$ C49, and GFP-PABPN1- $\triangle$ N160 constructs, which contain the cDNA encoding bovine PABPN1 subcloned in the pEGFP-C1 vector (BD Biosciences Clontech), were previously described (Calado et al. 2000a). GFP-PABPN1-ala17, GFP-PABPN1- $\Delta$ N113 and GFPPABPN1- $\Delta$ ala were subcloned from the eukaryotic pGM10 expression vector (Smith et al. 1999) into pEGFP-C1. All the cDNAs were initially cut with NdeI and the recessed 3' ends were filled in with Klenow. The linear plasmid was then cut with BamHI and the NdeI(filled)-BamHI fragment was purified. The pEGFP-C1 vector was cut with SmaI and BamHI and ligated to the cDNA fragment. The GFP-PABPN1- $\Delta 118-145$ mutant was constructed as follows. Using GFP-PABPN1-wt as template, we deleted the DNA stretch comprised between nucleotides 352 and 435 by two PCR reactions using primers GFP Fwd (CGAGAAGCGCGATCACATGGTCC) and PABPN1 Rev (P-CGGGTCCTCAATGGCGCCGTC) for PCR1 and primers GFP Rev (TTCAGGGGGAGGTGTGGGAGG TT) and PABPN1 Fwd (P-CAGATGAATATGAGTCCACCTCCG) for PCR2. The product obtained from PCR1 was subsequently digested with EcoRI originating a 382-base pair fragment spanning from the EcoRI site in the pEGFP polylinker to nucleotide 351 of PABPN1. The product generated by PCR2 was digested with BamHI to originate a 483-base-pair fragment spanning from nucleotide 436 of PABPN1 to the BamHI site in the pEGFP polylinker. The two PABPN1 fragments were then ligated to each other and were inserted into EcoRI/BamHI digested pEGFP C1. Plasmids containing point mutations in the coiled-coil domain of PABPN1 have been described in Kerwitz et al. (2003). The plasmid GFP-PABPN1-ala17 was digested in the unique XhoI site in the PABPN1 coding sequence and in the BamHI site at the $3^{\prime}$ end in the polylinker. The original XhoI-BamHI fragment was then replaced by the corresponding XhoI-BamHI DNA fragments with point mutations in the $\alpha$-helical domain of PABPN1. The same procedure was used to construct the GFP-PABPN1-ala17dm fusion. The GFP-PABPN1- $\Delta$ N50 and GFP-PABPN1- $\Delta$ N65 were subcloned from pDNRLib into pEGFP-C3 with EcoRI and KpnI.

Additionally, a normal human PABPN1 cDNA was obtained from the IMAGE. clone 4672226 and inserted in the $\mathrm{pTRE}_{2}$ hyg vector (Clontech) between the BamHI (filled in) and NheI (ligated to XbaI) cloning sites. A C-terminal hemaglutinin (HA) epitope was inserted by PCR using pTRE2hyg-PABPN1 as a template and the primers HA Fwd (GAGCCGGGACTGGTCGAGGGTGACC CG) and HA Rev (CGATCGATTTATGCATAATCAGGGACGTC GTATGGGTACATTCCGTAAGGGGAATACC), which contain restriction sites for BstEII and ClaI, respectively. The pTRE2hygPABPN1 plasmid was cut in the BstEII unique site and in the ClaI 
site in the polylinker originating a fragment containing the $3^{\prime}$ sequence of PABPN1 that was substituted by the digested PCR product. Next, the PABPN1-HA cDNA was subcloned into pcDNA3 vector using the KpnI and XhoI (ligated to SalI) restriction sites in the polylinker.

All DNA constructs were verified by sequencing. Final DNA plasmids were purified using the QIAGEN plasmid DNA midi kit. Subconfluent HeLa and C2 cells were transfected using FuGENE6 (Roche Biochemicals) according to the manufacturer's instructions. Cells were analyzed at 16-24 hr after transfection.

\section{Fluorescence microscopy and photobleaching}

Fluorescent samples were analyzed using the laser scanning microscope LSM 510 (Carl Zeiss). For quantitative analysis, images were obtained from cells expressing normal or expanded GFPPABPN1 using the same acquisition settings. From each experiment, average fluorescence intensities were estimated for 10 randomly selected cells. FRAP and FLIP experiments were performed as described (Calapez et al. 2002).

\section{Immunoelectron microscopy}

C2 cells expressing GFP-PABPN1 were fixed in formaldehyde and embedded in Lowicryl K4 M as previously described (Lafarga et al. 2002). Ultrathin sections on gold grids were sequentially incubated with anti-GFP antibody (Roche Biochemicals) and an appropriate secondary antibody conjugated to 5-nm gold particles (BioCell). The samples were examined with an EM208 electron microscope (Philips, Eindhoven) operated at $60 \mathrm{kV}$.

\section{SDS-PAGE and immunoblotting}

SDS-PAGE and Western blotting were performed as previously described (Almeida et al. 1998). Cells were washed twice in PBS, mixed with SDS sample buffer and Benzonase endonuclease (Sigma-Aldrich) and boiled. Proteins were separated by SDSPAGE on $10 \%$ polyacrylamide minigels (BioRad Laboratories) and electroblotted to a nitrocelulose membrane. Membranes were washed in PBS, blocked with PBS 5\% low fat milk for at least $1 \mathrm{hr}$ and incubated with specific primary antibodies diluted in PBS $2.5 \%$ low fat milk at $4^{\circ} \mathrm{C}$ overnight. Membranes were then washed $3 \times 15$ min in PBS-Tw 2.5\% low fat milk, incubated with appropriated secondary antibodies conjugated with horseradish peroxidase (BioRad Laboratories) and developed using a chemiluminescence reaction (ECL; Amersham Buchler GmbH).

\section{ACKNOWLEDGMENTS}

We thank E. Wahle (Martin-Luther-Universität Halle, Germany) for critical reading of the manuscript and for providing PABPN1 reagents. We further acknowledge our colleague Célia Carvalho for help with FISH experiments. We are also grateful to W. Keller (University of Basel, Switzerland) for generously providing antibodies directed against CPSF subunits and poly(A) polymerase; J. Manley (Columbia University, New York, USA) for anti-CstF50 monoclonal antibody; G. Dreyfuss (University of Pennsylvania, Philadelphia, USA) for mAbs 4F4 and 9H10 (anti-hnRNPA1); T.
Maniatis (Harvard University, USA) for mAb SC35; J. Castaño (Autonomous University of Madrid, Spain) for anti-proteasome antibodies; and A. Lamond (University of Dundee, UK) for poly(U) riboprobe.

This study was supported by grants from "Fundação para a Ciência e Tecnologia, POCTI/36547/MGI/00" (Portugal) and the European Commission "QLG2-CT-2001-01673". J.P.T. and P.C. are fellows from FCT (BD/2953/2000 and BD/4865/2001).

Received October 22, 2004; accepted January 21, 2005.

\section{REFERENCES}

Almeida, F., Saffrich, R., Ansorge, W., and Carmo-Fonseca, M. 1998. Microinjection of anti-coilin antibodies affects the structure of coiled bodies. J. Cell Biol. 142: 899-912.

Arribas, J., Arizti, P., and Castano, J.G. 1994. Antibodies against the C2 $\mathrm{COOH}$-terminal region discriminate the active and latent forms of the multicatalytic proteinase complex. J. Biol. Chem. 269: 1285812864.

Bao, Y.P., Cook, L.J., O’Donovan, D., Uyama, E., and Rubinsztein, D.C. 2002. Mammalian, yeast, bacterial, and chemical chaperones reduce aggregate formation and death in a cell model of oculopharyngeal muscular dystrophy. J. Biol. Chem. 277: 12263-12269.

Barabino, S.M., Hubner, W., Jenny, A., Minvielle-Sebastia, L., and Keller, W. 1997. The 30-kD subunit of mammalian cleavage and polyadenylation specificity factor and its yeast homolog are RNAbinding zinc finger proteins. Genes \& Dev. 11: 1703-1716.

Bear, D.G., Fomproix, N., Soop, T., Bjorkroth, B., Masich, S., and Daneholt, B. 2003. Nuclear poly(A)-binding protein PABPN1 is associated with RNA polymerase II during transcription and accompanies the released transcript to the nuclear pore. Exp. Cell Res. 286: 332-344.

Becher, M.W., Kotzuk, J.A., Davis, L.E., and Bear, D.G. 2000. Intranuclear inclusions in oculopharyngeal muscular dystrophy contain poly(A) binding protein 2. Ann. Neurol. 48: 812-815.

Berciano, M.T., Villagra, N.T., Ojeda, J.L., Navascues, J., Gomes, A., Lafarga, M., and Carmo-Fonseca, M. 2004. Oculopharyngeal muscular dystrophy-like nuclear inclusions are present in normal magnocellular neurosecretory neurons of the hypothalamus. Hum. Mol. Genet. 13: 829-838.

Bienroth, S., Keller, W., and Wahle, E. 1993. Assembly of a processive messenger RNA polyadenylation complex. EMBO J. 12: 585-594.

Bochtler, M., Ditzel, L., Groll, M., Hartmann, C., and Huber, R. 1999. The proteasome. Annu. Rev. Biophys. Biomol. Struct. 28: 295-317.

Bossy-Wetzel, E., Schwarzenbacher, R., and Lipton, S.A. 2004. Molecular pathways to neurodegeneration. Nat. Med. 10 Suppl.: S2-9.

Brais, B., Bouchard, J.P., Xie, Y.G., Rochefort, D.L., Chretien, N., Tome, F.M., Lafreniere, R.G., Rommens, J.M., Uyama, E., Nohira, O., et al. 1998. Short GCG expansions in the PABP2 gene cause oculopharyngeal muscular dystrophy. Nat. Genet. 18: 164-167.

Braun, I.C., Rohrbach, E., Schmitt, C., and Izaurralde, E. 1999. TAP binds to the constitutive transport element (CTE) through a novel RNA-binding motif that is sufficient to promote CTE-dependent RNA export from the nucleus. EMBO J. 18: 1953-1965.

Calado, A. and Carmo-Fonseca, M. 2000. Localization of poly(A)binding protein 2 (PABP2) in nuclear speckles is independent of import into the nucleus and requires binding to poly(A) RNA. $J$. Cell Sci. 113(Pt 12): 2309-2318.

Calado, A., Kutay, U., Kuhn, U., Wahle, E., and Carmo-Fonseca, M. 2000a. Deciphering the cellular pathway for transport of poly(A)binding protein II. RNA 6: 245-256.

Calado, A., Tome, F.M., Brais, B., Rouleau, G.A., Kuhn, U., Wahle, E., and Carmo-Fonseca, M. 2000b. Nuclear inclusions in oculopharyngeal muscular dystrophy consist of poly(A) binding protein 2 aggregates which sequester poly(A) RNA. Hum. Mol. Genet. 9: $2321-2328$. 
Calapez, A., Pereira, H.M., Calado, A., Braga, J., Rino, J., Carvalho, C., Tavanez, J.P., Wahle, E., Rosa, A.C., and Carmo-Fonseca, M. 2002. The intranuclear mobility of messenger RNA binding proteins is ATP dependent and temperature sensitive. J. Cell Biol. 159: 795-805.

Chai, Y., Shao, J., Miller, V.M., Williams, A., and Paulson, H.L. 2002. Live-cell imaging reveals divergent intracellular dynamics of polyglutamine disease proteins and supports a sequestration model of pathogenesis. Proc. Natl. Acad. Sci. 99: 9310-9315.

Chen, Z., Li, Y., and Krug, R.M. 1999. Influenza A virus NS1 protein targets poly(A)-binding protein II of the cellular 3 '-end processing machinery. EMBO J. 18: 2273-2283.

Choi, Y.D. and Dreyfuss, G. 1984. Monoclonal antibody characterization of the $\mathrm{C}$ proteins of heterogeneous nuclear ribonucleoprotein complexes in vertebrate cells. J. Cell Biol. 99: 1997-1204.

Fan, X., Dion, P., Laganiere, J., Brais, B., and Rouleau, G.A. 2001. Oligomerization of polyalanine expanded PABPN1 facilitates nuclear protein aggregation that is associated with cell death. Hum. Mol. Genet. 10: 2341-2351.

Fornerod, M., Boer, J., van Baal, S., Jaegle, M., von Lindern, M., Murti, K.G., Davis, D., Bonten, J., Buijs, A., and Grosveld, G. 1995. Relocation of the carboxyterminal part of CAN from the nuclear envelope to the nucleus as a result of leukemia-specific chromosome rearrangements. Oncogene 10: 1739-1748.

Fu, X.D. and Maniatis, T. 1990. Factor required for mammalian spliceosome assembly is localized to discrete regions in the nucleus. Nature 343: 437-441.

Gama-Carvalho, M., Krauss, R.D., Chiang, L., Valcarcel, J., Green, M.R., Carmo-Fonseca, M. 1997. Targeting of U2AF65 to sites of active splicing in the nucleus. J. Cell Biol. 137: 975-987.

Görlach, M., Burd, C.G., and Dreyfuss, G. 1994. The mRNA poly(A)binding protein: Localization, abundance, and RNA-binding specificity. Exp. Cell Res. 211: 400-407.

Jenny, A., Hauri, H.P., and Keller, W. 1994. Characterization of cleavage and polyadenylation specificity factor and cloning of its 100kilodalton subunit. Mol. Cell. Biol. 14: 8183-8190.

Johnson, C.V., Singer, R.H., and Lawrence, J.B. 1991. Fluorescent detection of nuclear RNA and DNA: Implications for genome organization. Methods Cell Biol. 35: 73-99.

Kerwitz, Y., Kuhn, U., Lilie, H., Knoth, A., Scheuermann, T., Friedrich, H., Schwarz, E., and Wahle, E. 2003. Stimulation of poly(A) polymerase through a direct interaction with the nuclear poly(A) binding protein allosterically regulated by RNA. EMBO J. 22: $3705-3714$.

Kim, S., Nollen, E.A., Kitagawa, K., Bindokas, V.P., and Morimoto, R.I. 2002. Polyglutamine protein aggregates are dynamic. Nat. Cell Biol. 4: 826-831.

Krause, S., Fakan, S., Weis, K., and Wahle, E. 1994. Immunodetection of poly(A) binding protein II in the cell nucleus. Exp. Cell Res. 214: $75-82$.

Kühn, U. and Wahle, E. 2004. Structure and function of poly(A) binding proteins. Biochim. Biophys. Acta 1678: 67-84.

Kühn, U., Nemeth, A., Meyer, S., and Wahle, E. 2003. The RNA binding domains of the nuclear poly(A)-binding protein. J. Biol. Chem. 278: 16916-16925.

Lafarga, M., Berciano, M.T., Pena, E., Mayo, I., Castano, J.G., Bohmann, D., Rodrigues, J.P., Tavanez, J.P., and Carmo-Fonseca, M. 2002. Clastosome: A subtype of nuclear body enriched in $19 S$ and $20 \mathrm{~S}$ proteasomes, ubiquitin, and protein substrates of proteasome. Mol. Biol. Cell 13: 2771-2782.

Lamond, A.I. and Spector, D.L. 2003 Nuclear speckles: A model for nuclear organelles. Nat. Rev. Mol. Cell. Biol. 4: 605-612.

Lennon, G., Auffray, C., Polymeropoulos, M., and Soares, M.B. 1996.
The I.M.A.G.E. Consortium: An integrated molecular analysis of genomes and their expression. Genomics 33: 151-152.

Mengual, E., Arizti, P., Rodrigo, J., Gimenez-Amaya, J.M., and Castano, J.G. 1996. Immunohistochemical distribution and electron microscopic subcellular localization of the proteasome in the rat CNS. J. Neurosci. 16: 6331-6341.

Murthy, K.G. and Manley, J.L. 1995. The $160-\mathrm{kD}$ subunit of human cleavage-polyadenylation specificity factor coordinates pre-mRNA 3 '-end formation. Genes \& Dev. 9: 2672-2683.

Nemeth, A., Krause, S., Blank, D., Jenny, A., Jeno, P., Lustig, A., and Wahle, E. 1995. Isolation of genomic and cDNA clones encoding bovine poly(A) binding protein II. Nucleic Acids Res. 23: 40344041.

Orr, H.T. 2001. Beyond the Qs in the polyglutamine diseases. Genes Dev. 15: 925-932.

Reits, E.A. and Neefjes, J.J. 2001. From fixed to FRAP: Measuring protein mobility and activity in living cells. Nat. Cell Biol. 3: E145147.

Rodrigues, J.P., Rode, M., Gatfield, D., Blencowe, B.J., Carmo-Fonseca, M., and Izaurralde, E. 2001. REF proteins mediate the export of spliced and unspliced mRNAs from the nucleus. Proc. Natl. Acad. Sci. 98: 1030-1035.

Scheuermann, T., Schulz, B., Blume, A., Wahle, E., Rudolph, R., and Schwarz, E. 2003. Trinucleotide expansions leading to an extended poly-L-alanine segment in the poly (A) binding protein PABPN1 cause fibril formation. Protein Sci. 12: 2685-2692.

Smith, J.J., Rucknagel, K.P., Schierhorn, A., Tang, J., Nemeth, A., Linder, M., Herschman, H.R., and Wahle, E. 1999. Unusual sites of arginine methylation in Poly(A)-binding protein II and in vitro methylation by protein arginine methyltransferases PRMT1 and PRMT3. J. Biol. Chem. 274: 13229-13234.

Stenoien, D.L., Mielke, M., and Mancini, M.A. 2002. Intranuclear ataxin 1 inclusions contain both fast- and slow-exchanging components. Nat. Cell Biol. 4: 806-810.

Takagaki, Y., Manley, J.L., MacDonald, C.C., Wilusz, J., and Shenk, T. 1990. A multisubunit factor, CstF, is required for polyadenylation of mammalian pre-mRNAs. Genes \& Dev. 4: 2112-2120.

Taylor. J.P., Hardy, J., and Fischbeck, K.H. 2002. Toxic proteins in neurodegenerative disease. Science 296: 1991-1995.

Uyama, E., Tsukahara, T., Goto, K., Kurano, Y., Ogawa, M., Kim, Y.J., Uchino, M., and Arahata, K. 2000. Nuclear accumulation of expanded PABP2 gene product in oculopharyngeal muscular dystrophy. Muscle Nerve 23: 1549-1554.

Wahle, E. 1991a. A novel poly(A)-binding protein acts as a specificity factor in the second phase of messenger RNA polyadenylation. Cell 66: 759-768.

- 1991b. Purification and characterization of a mammalian polyadenylate polymerase involved in the $3^{\prime}$ end processing of messenger RNA precursors. J. Biol. Chem. 266: 3131-3139.

. 1995. Poly(A) tail length control is caused by termination of processive synthesis. J. Biol. Chem. 270: 2800-2808.

Wahle, E., Lustig, A., Jeno, P., and Maurer, P. 1993. Mammalian poly(A)-binding protein II. Physical properties and binding to polynucleotides. J. Biol. Chem. 268: 2937-2945.

White, J. and Stelzer, E. 1999. Photobleaching GFP reveals protein dynamics inside live cells. Trends Cell Biol. 9: 61-65.

Zirbel, R.M., Mathieu, U.R., Kurz, A., Cremer, T., and Lichter, P. 1993. Evidence for a nuclear compartment of transcription and splicing located at chromosome domain boundaries. Chromosome Res. 1: 93-106.

Zoghbi, H.Y. and Orr, H.T. 2000. Glutamine repeats and neurodegeneration. Annu. Rev. Neurosci. 23: 217-247. 

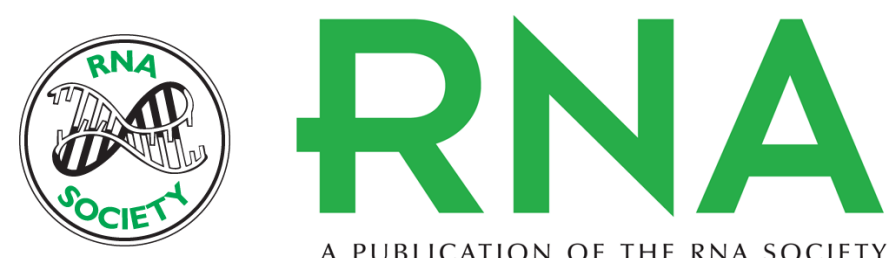

A PUBLICATION OF THE RNA SOCIETY

\section{In vivo aggregation properties of the nuclear poly(A)-binding protein PABPN1}

JOÃO PAULO TAVANEZ, PATRICIA CALADO, JOSÉ BRAGA, et al.

RNA 2005 11: 752-762

References This article cites 52 articles, 26 of which can be accessed free at:

http://rnajournal.cshlp.org/content/11/5/752.full.html\#ref-list-1

\section{License}

Email Alerting Receive free email alerts when new articles cite this article - sign up in the box at the Service top right corner of the article or click here. 